\title{
Navigating Change: How Outreach Facilitators Can Help Clinicians Improve Patient Outcomes
}

\author{
Dianne Laferriere, RN, BScN, Clare Liddy, MDMSc, CCFP, Kate Nash, MSc, and \\ William Hogg, MClSC MDCM, CCFP
}

Objective: The objective of this study was to describe outreach facilitation as an effective method of assisting and supporting primary care practices to improve processes and delivery of care.

Methods: We spent 4 years working with 83 practices in Eastern Ontario, Canada, on the Improved Delivery of Cardiovascular Care through the Outreach Facilitation program.

Results: Primary care practices, even if highly motivated, face multiple challenges when providing quality patient care. Outreach facilitation can be an effective method of assisting and supporting practices to make the changes necessary to improve processes and delivery of care. Multiple jurisdictions use outreach facilitation for system redesign, improved efficiencies, and advanced access.

Conclusions: The development and implementation of quality improvement programs using practice facilitation can be challenging. Our research team has learned valuable lessons in developing tools, finding resources, and assisting practices to reach their quality improvement goals. These lessons can lead to improved experiences for the practices and overall improved outcomes for the patients they serve. (J Am Board Fam Med 2012;25:232-237.)

Keywords: Outreach Facilitator, Patient Outcomes, Practice Facilitation, Primary Health Care

A primary care group practice comprising 5 family physicians and multiple allied health personnel was located over 3 sites. It was primarily an urban, English-speaking practice but had patients of various ethnicities. Physicians had a rostered patient load of approximately 1300 each and had access to electronic medical records (EMRs) and Internet resources. The practice had some processes in place for diabetes management and had met its recommended target of measuring 2 glycosylated hemoglobin (HbA1c) levels each for their diabetic patients within a year. However, there remained a

This article was externally peer reviewed.

Submitted 7 June 2011; revised 31 December 2011; accepted 5 January 2012.

From the C.T. Lamont Primary Health Care Research Centre, Élisabeth Bruyère Research Institute, Ottawa, Ontario, Canada.

Funding: The IDOCC initiative is funded through the Champlain Cardiovascular Disease Prevention Network, the Ottawa Hospital Academic Medical Organization, and the Ontario Ministry of Health and Long Term Care.

Conflict of interest: none declared.

Corresponding author: Clare Liddy, C.T. Lamont Primary Health Care Research Centre, Élisabeth Bruyère Research Institute, 43 Bruyère St., Ottawa, Ontario, Canada K1N 5C8 (E-mail: cliddy@bruyere.org). group of patients with $\mathrm{HbA1c}$ values who were not at target, and the team was unsure about how they could address this difficulty. They wondered how they were performing in other areas and what might need to be changed. What should they do?

Primary care practices face multiple challenges. Patient care is increasingly complex, practice models are changing, and time and money are in short supply. Within this context, making changes to improve patient care can be a daunting task. Often, clinicians have trouble defining what they want to accomplish, are unsure of how to institute changes, or may not know how to evaluate whether the changes have improved the care they deliver to their patients. Therefore, one method that has arisen to help practices make these quality improvements (QIs) is the use of an outreach facilitator $(\mathrm{OF})$. An $\mathrm{OF}$ is a health care professional with expertise in organizational change management who can lead and support health care providers down the path of change. Using tools such as chart audits and feedback, OFs help practice physicians recognize challenges and identify goals. Then, by working collaboratively, the $\mathrm{OF}$ and the physicians can develop plans to accomplish these goals. 
Table 1. Examples of Programs Using Outreach Facilitation

\begin{tabular}{ll}
\hline IMPACT BC & http://www.impactbc.ca/ \\
Quality Improvement \& Innovation Partnership, Ontario & http://www.qiip.ca/ \\
Health Quality Ontario & http://www.ohqc.ca/en/index.php \\
AIM Alberta & http://www.albertaaim.ca/ \\
Quality Diabetes Collaborative- Capital District Health Authority, & www.cdha.nshealth.ca \\
$\quad$ Nova Scotia & http://www.gov.mb.ca/health/mpan/projects.html \\
Pursuing Excellence: Manitoba Patient Access Network &
\end{tabular}

Practices vary considerably in size, organizational culture (eg, paper-based or computer-based practices), and payment models. Therefore, successful facilitation needs to be wide-ranging, multifaceted, and individually tailored to meet the needs of each specific practice. A recent meta-analysis has shown that primary care physicians are almost 3 times more likely to adopt evidence-based guidelines when they are supported by an OF. ${ }^{1}$ Currently, there are multiple facilitation programs across Canada and the United States that are engaged in system redesign, improvement of efficiencies, and advanced access (see Table 1). The goal of this article is to describe a project in which we employed the use of outreach facilitators among 83 practices and to elucidate the barriers we encountered and the lessons learned.

\section{Methods}

\section{Improved Delivery of Cardiovascular Care Through Outreach Facilitation}

Our project, the Improved Delivery of Cardiovascular Care (IDOCC) through Outreach Facilitation, is a QI program that aims to improve the care provided to patients who have or are at high risk of developing cardiovascular disease. We recruited a total of 83 practices and employed a step-wedge design over 3 years of working with these practices. Each of our facilitators visited, on average, 12 to 15 practices monthly during the first year (less frequently during the second) to support the changes necessary to improve the practices' overall delivery of guideline-specific, evidence-based care.

\section{Audit and Feedback}

Our first step was to use audit and feedback (A\&F), a powerful tool that has been shown to have a modest but significant positive effect on quality outcomes (see Table 2). ${ }^{2,3}$ A\&F describes processes and outcomes at a particular moment in time. Points of data from a sample of appropriate patient charts are gathered and compiled from an individual provider or a group of providers. This data also can be compared with the mean of all practices. The information is then presented during a neutral feedback session to allow the practice members to assess their performances. A practice may simultaneously have areas of above- and below-average performance when compared with the mean-an outcome of the audit that may surprise them.

Table 2. The Improved Delivery of Cardiovascular Care Chart Audit and Feedback Process

\begin{tabular}{|c|c|}
\hline Purpose & To get a "snapshot" of care processes and/or outcomes at a moment in time and present this picture to a practice. \\
\hline Step 1 & $\begin{array}{l}\text { Determine which indicators are wanted; in our case, CVD risk factor indicators. So, we decided to include BP } \\
\text { measurements, aspirin recommendations, LDL measurements, ACE inhibitor use, smoking cessation } \\
\text { recommendations, and eGFR and ACR levels. }\end{array}$ \\
\hline Step 2 & Select sample of patients from entire patient roster \\
\hline Step 3 & $\begin{array}{l}\text { Review each chart to ensure eligibility and search for documented indicators within the time span. For example, } \\
\text { for patients with a diagnosis of hypertension, the audit will reveal if } 2 \mathrm{BP} \text { measurements were taken and } \\
\text { documented within the last } 12 \text { months. }\end{array}$ \\
\hline Step 4 & $\begin{array}{l}\text { OF receives data and organizes it for each practice by disease and/or risk factor and presents it to the practice in a } \\
\text { neutral, nonjudgemental way. This can show comparison with mean, maximum, and minimum values achieved } \\
\text { over a range of practices to give an overview of current processes and outcomes of care. }\end{array}$ \\
\hline Step 5 & $\begin{array}{l}\text { Building on this opportunity, current guidelines of care are discussed, practice goals are explored, and a consensus } \\
\text { among team members is reached. }\end{array}$ \\
\hline
\end{tabular}

ACE, angiotensin-converting enzyme; ACR, albumin:creatinine ratio; BP, blood pressure; CVD, cardiovascular disease; eGFR, estimated glomerular filtration rate; LDL, low-density lipoprotein; OF, outreach facilitator. 
Throughout the feedback and subsequent discussions, the OF can help gain consensus from the various members of the team to allow them to explore their vision of their ideal practice and to help them set goals for change. A\&F also can provide target outcome measures and may be used to evaluate changes in processes for effectiveness.

\section{Results}

Regarding the case study that opened this article, the $A \& F$ revealed some areas that piqued the team's interest. We will use this case study to demonstrate specific examples of practice improvements the OF supported; then we will describe the lessons learned from this study to illustrate varying roles within a practice the OF can play.

\section{Case Study Example}

Aspirin Use

Recent changes in guidelines for aspirin use and the availability of this medication over the counter has resulted in inconsistent prescribing patterns and documentation among practice providers. In addition, adherence to guidelines of care for hypertension (as measured by blood pressure [BP]) and diabetes care (as measured by HbA1c levels) was lower than expected. Consequently, the OF suggested and discussed possible approaches to address these concerns. First, she provided current aspirinuse recommendations for patients with diabetes and cardiovascular disease in general. The team felt that a lack of consistent aspirin notation in the EMR was a barrier to implementing appropriate medication recommendations and that improving this notation would be an easy change to make. The staff discussed and agreed on specific terminology, embedded an alert for aspirin within the EMR, and decided to write prescriptions for aspirin, even if patients would purchase it over the counter, to ensure appropriate use.

\section{Improving Hypertension Control}

In the case study, the A\&F had revealed that some patients' BPs were not being adequately monitored and controlled. The majority of the physicians felt that they should be able to achieve targets given the resources that they had available. There also was a feeling among the team that patients needed to take more responsibility for their own health and follow-up appointments. Being cognizant of the dif- ferent views regarding patient responsibility, the OF presented a systematic review to show how recall works to improve $\mathrm{BP}$ control. ${ }^{4}$ In preparation for a recall system, a registry would need to be established. In the meantime, the practice decided that it would use the team members more effectively, develop a BP protocol, and apply for budgetary funds to purchase a BP Tru machine. In a subsequent meeting, the $\mathrm{OF}$ followed up on the topic of a recall system. The pharmacist agreed to lead the work and to set the time frames. Until a registry could be established, the team would run a sample search of patients with hypertension who were not at target. The OF introduced the Plan, Do, Study, Act (PDSA) QI tool. The primary care group implemented changes in processes to identify patients, to monitor BP every visit, and to record every $\mathrm{BP}$ in the EMR. Within several months they were able to distinguish those patients who were being managed well from those who were falling between the cracks. Those who were less well-managed were thereafter called back into the office more frequently to get their BP under control.

\section{Revisiting Hypertension and HbA1c Levels}

Toward the end of the first year of participation, our original case study practice was visited again by the OF during a scheduled visit. The OF wanted to close the loop on the hypertension management QI and brought up the subject of the registry. It seemed that although the small pilot project had worked to a degree, no further action had been taken to establish a registry and work toward those BP target outcomes in a consistent way. After talking about their original plan, and with encouragement from the OF, the team was willing to try again. In addition, talks revolved around an initial area of interest in improving HbA1c outcomes for those patients who were not well managed. The group had put a lot of effort into improving their processes for diabetes care and developing a recall system, but improving outcomes needed another approach. They decided to set up a diabetes group comprising the diabetes team, clinical, and administrative staff, with the clinical coordinator as the leader. They agreed on a step-wise strategy in which they would rationalize referrals to community diabetes education programs to avoid duplication of services, pilot a self-management program within the practice, and hold a training day to finalize their strategy. The OF put them in touch with another team that was willing to share their experiences with 
setting up a diabetes self-management program. It was hoped that these actions would help some of those patients reach better $\mathrm{HbA1c}$ targets.

\section{Lessons Learned}

\section{Practice Culture}

Practice culture is important to consider when implementing a QI initiative. Autonomy, collegiality, and technological biases are just some aspects of a culture that influence the style of work and adaptation to or acceptance of change. In one paperbased practice, the OF proposed a system for recalling patients with diabetes or hypertension by using a simple file card registry that would only involve a few phone calls a month. By the next visit, the practice had devised a simple system using a dummy billing code. This busy practice put an effective system in place with few resources. They also needed some educational resources for patients, but space was limited and the staff did not want the facilitator to bring paper resources that were difficult to store. After several discussions, it was agreed that small, wallet-sized cards with only a few references to pertinent web sites would be useful, and the team would provide their patients with these cards. Changes had to be practical and tailored to the practice's requirements and culture.

\section{Evaluating Effectiveness}

Another of the many lessons learned was that although a practice may be organized, with established programs to improve chronic disease management processes and support for patients in managing their conditions, issues arise with which an $\mathrm{OF}$ can assist. We worked with a primary care group practice, recognized as a care leader, that al-

Figure 1. Planning and evaluation form.

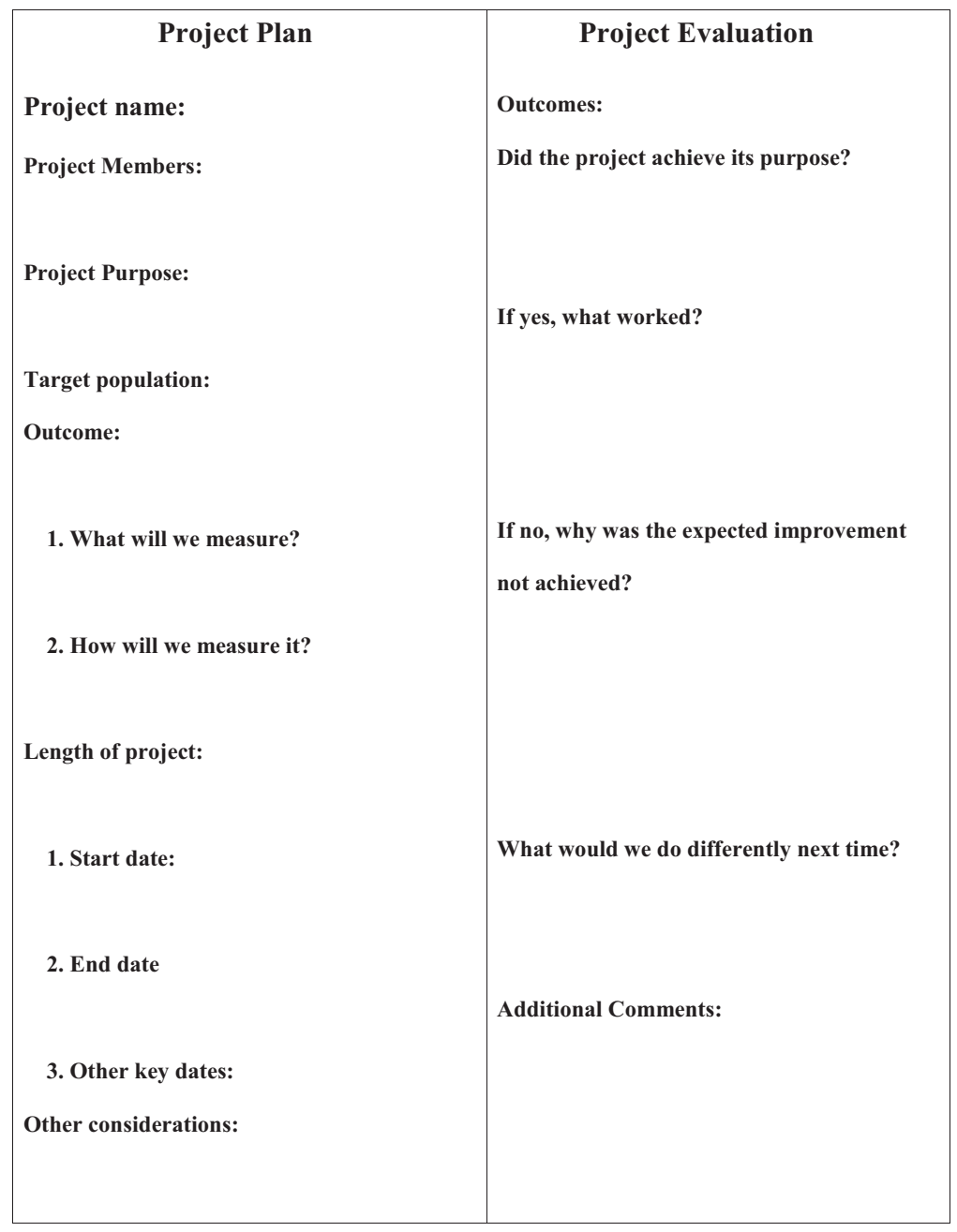


Table 3. An Example of Utilizing Plan, Do, Study, Act Cycle

\begin{tabular}{ll}
\hline Plan & To improve recording of BP of hypertensive patients \\
\hline Do & Receptionist to flag charts for every hypertensive patient who comes in for regular appointment \\
& Nurse or physician to record every patient's BP for one day \\
Study & Review charts for that day to verify that BP has been recorded \\
& Two patients did not have BPs recorded because health care provider made notations at end of visit and could not \\
& remember the BP \\
Act & Will repeat another day and health care providers will record BP immediately instead of waiting until end of visit \\
& Reassess at end of next day
\end{tabular}

$\mathrm{BP}$, blood pressure.

ready had patient educational and monitoring programs and had initiated many projects using the data provided by their disease registries. After the first few meetings, the $\mathrm{OF}$ wondered whether she had anything to contribute to the team. It became apparent that it was difficult for them to gauge whether an approach had been effective. The OF collated various QI tools and designed a tailored tool that could help them define their objectives and outcomes, plan their intervention, and then evaluate it. She presented it to the team at the next meeting. The tool is now being used routinely and seems to be focusing the team toward effective evaluation of outcomes (see Figure 1).

\section{Reducing Inefficiencies}

OFs can work with practices to identify areas of waste, redundancies, and inefficiencies and to examine the full scope of roles using proven methods such as LEAN and Six Sigma. ${ }^{5}$ Process analyses can be made with Ishekawa diagrams and process mapping strategies. ${ }^{6,7}$ An effective tool that is widely used is the PDSA cycle (see Table 3). Once a practice has decided on process or outcome goals, PDSA cycles are a simple way to implement changes and evaluate results. Small changes in processes can be made and then immediately studied for outcomes within a short time frame. If the changes result in progress toward the goals, then actions can be continued. If not, alterations can be made and the changes evaluated. Changes do not need to be complex. Simple things such as reminders in an EMR or paper chart are often very effective.

\section{Community Resources}

Accurate listings and knowledge of community resources is another important component in the delivery of quality care. Community organizations have been funded through municipal, provincial/ state, and federal jurisdictions, but practices often lack an awareness of these local resources. An OF can update and disseminate these resources for maximum use by practitioners and patients alike, resulting in improved health and self-management.

\section{Conclusions}

Changes in primary care will continue to occur, as will efforts to improve quality. The IDOCC program has revealed and confirmed some key lessons in change management. Effective outreach facilitation needs to be wide ranging, multifaceted, and tailored to the needs of the individual practice. Delays and uncertainties are commonplace, so $\mathrm{A} \& \mathrm{~F}$ and goal setting are important steps in any QI initiative. They clarify baseline positions, and the practice can explore its ideal vision of care delivery and target achievement. There are many QI tools that already exist (see Table 4). There is no need to reinvent the wheel, but tailoring a tool to a specific practice culture can improve its efficacy considerably. Enhancing a practice's knowledge of local

\section{Table 4. Examples of Useful Tools That Can Be Utilized in Outreach Facilitation}

Audit and feedback

Best practice guidelines for care

PDSA cycles

Up-to-date listing of community resources

CVD flow sheets

Streamlined processes for referral to specialists

Patient self-management tools

Ask a specialist question and answer processes

Networks and sharing among practices

Tailored development of other QI tools

CVD, cardiovascular disease; PDSA, Plan, Do, Study, Act; QI, quality improvement. 
1. Hire facilitators who have the qualities to work with various providers and provide training in QI principles and change management.

2. Use audit and feedback to establish baseline and give motivation.

3. Employ a variety of tools such as Plan, Do, Study, Act; community resources and programs; and evaluation plans.

4. Tailor techniques and tools to specific practice needs.

5. Assist with enhanced access to specialists where possible.

6. Close the loop; revisit earlier work and evaluate progress, hurdles, and plans.

7. Celebrate small successes and build on them to progress.

QI, quality improvement.

community resources is a good way to improve referrals, and use of educational programs can increase patient self-management.

We have found that some practices are reluctant to introduce any change without evidence that it will improve patient outcomes. For example, though they may accept that waist circumference measurement is predictive of disease, they want to see the evidence that performing these measurements will change their patients' outcomes. They challenge the OFs to find the evidence and rationale for suggested approaches before making investments in time and effort.

\section{Outreach Facilitators and Change}

The skills an OF must possess to provide a practice with the opportunities, resources, and encouragement to succeed in its chosen QI goals include knowledge of primary care, change management and QI principles, evidence-based guidelines, and knowledge translation theory. An OF must have strong organizational and communication abilities and be flexible and neutral in their approach. When the skills and knowledge of an OF are aligned with the culture and readiness of a practice, change can occur. An OF can help to gain consensus, to build trust, to set goals, and to revisit issues to close the loop and assess the effectiveness of actions. If the actions taken were not effective, the $\mathrm{OF}$ can then engage practices to explore other measures to accomplish their goals. All these factors are key com- ponents for change to occur (see Table 5). In IDOCC, we have found that improving quality in the delivery of care has not always been easy; however, with the help of an OF, primary care practices can navigate the way as smoothly and as quickly as possible.

\section{References}

1. Baskerville NB, Liddy C, Hogg W. Systematic review and meta-analysis of practice facilitation within primary care settings. Ann Fam Med 2012;10:63-74.

2. Jamtvedt G, Young, JM, Kristoffersen DT, O'Brien MA, Oxman AD. Audit and feedback: effects on professional practice and health care outcomes. Cochrane Database Syst Rev 2006;2:CD000259.

3. Hysong S. Meta-analysis: audit and feedback features impact effectiveness on care quality. Med Care 2009; 47:356-63.

4. Glynn LG, Murphy AW, Smith SM, Schroeder K, Fahey $\mathrm{T}$. Interventions used to improve control of blood pressure in patients with hypertension. Cochrane Database Syst Rev 2010;3:CD005182.

5. Varkey P, Reller MK, Resar RK. Basics of quality improvement in health care. Mayo Clin Proc 2007; $82: 735-9$.

6. Fishbone diagram. Available at: http://www.improhealth. org/fileadmin/Documents/Improvement_Tools/ Fishbone_diagram.pdf. Accessed August 11, 2011.

7. NHS Institute for Innovation and Improvement. Process mapping: an overview. 2008. Available at http://www. institute.nhs.uk/quality_and_service_improvement_ tools/quality_and_service_improvement_tools/process_ mapping_-_an_overview.html. Accessed August 11, 2011. 